\title{
Overcoming Cost Implications of Mutational Analysis in Patients with Gastrointestinal Stromal Tumors: A Pragmatic Approach
}

\author{
Patrick Schöffski ${ }^{a, b} \quad$ Agnieszka Wozniak $^{c}$ Oliver Schöffski ${ }^{d}$ Liesbet van Eycken ${ }^{\mathrm{e}}$ \\ Maria Debiec-Rychter ${ }^{f}$ \\ a Department of General Medical Oncology, Leuven Cancer Institute, Leuven, Belgium; \\ ${ }^{b}$ Department of Oncology, Laboratory of Experimental Oncology, KU Leuven, Leuven, Belgium; \\ ${ }^{\mathrm{c}}$ Laboratory for Experimental Oncology, Department of Oncology, KU Leuven, Leuven, Belgium; \\ ${ }^{\mathrm{d}}$ Lehrstuhl für Gesundheitsmanagement, Friedrich-Alexander-Universität (FAU) Erlangen-Nürnberg, Nuremberg, Germany; \\ e Belgian Cancer Registry, Sint-Joost-ten-Node, Belgium; \\ ${ }^{f}$ Department of Human Genetics, KU Leuven and University Hospital Leuven, Leuven, Belgium
}

\section{Keywords}

Biomarker · Costs · Gastrointestinal stromal tumor . Tyrosine kinase $\cdot$ Kit $\cdot$ Platelet-derived growth factor receptor alpha - Mutational analysis - Imatinib .

Sunitinib · Regorafenib

\section{Summary}

Background: Genetic analysis of tissue derived from patients with advanced gastrointestinal stromal tumors (GISTs) is not uniformly applied on a national and international level, even though mutational data can provide clinically relevant prognostic and predictive information, especially in patients qualifying for treatment with expensive targeted agents. Methods: The current article describes the rationale for genetic testing of GIST tissue, looks at financial implications associated with such analysis and speculates on potential cost savings introduced by routine mutational testing and tailored use of tyrosine kinase inhibitors based on genotyping. This work is based on a hypothetical analysis of epidemiological data, drug costs, reimbursement criteria and market research figures. Results: The cost burden for routine genotyping of important genes in GISTs, especially in patients at high risk for relapse after primary surgery and in advanced, inoperable metastatic disease, is relatively low. The early identification of GISTs with primary resistance mutations should be the basis for personalized GIST treatment and reimbursement of drugs. As illustrated by Belgian figures, the exclusive use of a drug such as imatinib in patients who are likely to benefit from the agent based on genetic information can lead to significant cost savings, which outweigh the costs for testing. Conclusions: Mutational analysis of GIST should be considered early in all patients at risk for relapse after curative surgery and in the case of advanced, inoperable, metastatic disease. The costs for the actual genotyping should not be used as an argument against profiling of the tumor. The adjuvant and palliative systemic treatment of GISTs should be personalized based on the genotype and other known prognostic and predictive factors. Reimbursement criteria for essential agents such as imatinib should be adapted accordingly.

(c) 2016 S. Karger GmbH, Freiburg

\section{Introduction}

Gastrointestinal stromal tumors (GISTs) are the most common mesenchymal neoplasms occurring in the gastrointestinal tract. Most of these tumors are characterized by activating mutations in either the Kit gene or the PDGFRA (platelet-derived growth factor receptor alpha) gene, which result in constitutional, ligand-independent activation of the respective receptor tyrosine kinases (RTKs) and their downstream signaling pathways, leading to uncontrolled tumor cell proliferation [1].

The most common primary mutations in GISTs involve exon 11 of the Kit gene, encoding a juxtamembrane domain of the re-

\section{KARGER}

(c) 2016 S. Karger GmbH, Freiburg
Prof. Dr. Patrick Schöffski, MPH

Department of General Medical Oncology, Leuven Cancer Institute

Department of Oncology, Laboratory of Experimental Oncology, KU Leuven

Herestraat 49, 3000 Leuven, Belgium

patrick.schoffski@ uzleuven.be 
ceptor. This genetic change interferes with the autoinhibitory function of the receptor and RTK activation. The second most frequent mutation occurs in Kit exon 9, encoding the extracellular domain of the receptor, which is responsible for Kit ligand binding [2].

The dependence of GIST cells on oncogenic Kit/PDGFRA proteins makes this tumor an attractive target for the use of selective tyrosine kinase inhibitors (TKIs). Imatinib mesylate, a potent antagonist of mutated Kit, is highly effective in locally advanced, metastatic and inoperable GIST [3]. Imatinib competes with adenosine triphosphate (ATP) in binding to the receptor and, as a consequence, blocks the downstream signaling pathways.

It is known that the response to imatinib depends on the type of the initial Kit/PDGFRA mutation. Higher response rates and longer median overall survival have been observed in GIST patients with a Kit exon 11 mutation, compared to Kit exon 9-mutated GISTs. The latter patients require higher imatinib doses to achieve optimal benefit from the oral treatment [4].

During the course of therapy, the majority of patients treated with TKIs develop secondary resistance to the drug and they ultimately progress on treatment. These genetic changes are almost exclusively found in exons coding for the ATP binding pocket domain of the Kit receptor (tyrosine kinase 1 (TK1), encoded by exons 13 and 14) and in the kinase activation loop domain (TK2, encoded by exons 17 and 18) [5].

Mutations in the TK1 domain directly prevent imatinib binding to the RTK. Sunitinib, a multi-target TKI, can still inhibit these and other malignant clones and is the approved second-line standard of care for patients with imatinib-refractory disease [6].

Mutations in the TK2 domain induce a switch to the active conformation of the RTK and generally render GIST cells resistant to both imatinib and sunitinib [7]. In the clinic, sunitinib treatment results in prolonged progression-free survival in patients carrying secondary mutations in the TK1 domain, while no clinical benefit is observed in patients with mutations in the TK2 domain [8].

Regorafenib is a multi-target TKI that was recently approved as a third-line treatment for GIST patients failing both imatinib and sunitinib, with good efficacy against most mutations in the Kit TK2 domain. But some mutations in TK2, e.g. p.D816V, are generally resistant to all commercially available TKIs including this thirdgeneration agent [9].

Primary resistance of GISTs to imatinib at initial diagnosis is an uncommon finding but still affects $8-10 \%$ of all treated patients. Primary resistance can be the result of a PDGFRA p.D842V mutation. This mutation renders the tumors completely resistant to imatinib $[10,11]$. About 1 in 5 gastric GISTs carries a p.D842V mutation at diagnosis, and the stomach is one of the most common primary sites of GISTs. However, it has to be highlighted that these mutants are generally biologically less aggressive than Kit mutants. It is also important to know that, in advanced tumors, the incidence of PDGFRA gene-mutated GISTs is rather low. Combining data from a number of clinical trials, the incidence of PDGFRA mutations in advanced GISTs is believed to be in the range of $2-4 \%[12,13]$.

GISTs can also be driven by other, Kit/PDGFRA-independent mechanisms. Mutations of the Ras pathway and defects in the $S D H$ (succinate dehydrogenase) gene can induce a similar phenotype. Tumors that lack abnormalities of the Kit, PDGFRA, SDH, or Ras signaling pathways are now commonly described as 'quadruple wild-type GISTs'. The sensitivity of such tumors to established TKIs has not been studied systematically, but it is unlikely that such cases will respond well to highly specific Kit/PDGFRA inhibitors, even though they may resemble classical GISTs in terms of clinical presentation and morphology [14].

Among the approved TKIs for GISTs, imatinib mesylate is the only agent that is also routinely used for adjuvant therapy after complete resection of the primary tumor in patients at increased risk for relapse. Imatinib has been available for adjuvant treatment since 2002 (United State Food and Drug Administration approval) and has proven to be a successful therapy in a number of largescale randomized trials. The current adjuvant standard of care is to treat GIST patients at high risk for relapse for 3 years after initial curative surgery with imatinib, with the intention to delay the occurrence of a relapse or even to eradicate microscopic residual disease and cure the patient. At present, ongoing studies are evaluating longer durations of imatinib treatment in this setting, and some physicians already advocate life-long use of the drug in individual patients at highest risk of relapse. A similar approach is taken for patients with pre- or intraoperative rupture of the primary GIST with a high risk of micrometastatic spread of the disease. These patients are usually grouped together with patients with metastatic GIST [15].

While initial adjuvant imatinib trials did not routinely assess the presence or absence of an imatinib-resistant genotype prior to patient entry, it is generally believed that patients with tumors driven by PDGFRA p.D842V or other, less common primary resistance mutations cannot benefit from the adjuvant therapy with imatinib [16].

In a database of 1,500 GIST cases in which the Department of Human Genetics at the University Hospitals in Leuven performed mutational analysis, the incidence of the PDGFRA mutation was 6\% (M.D.-R., personal communication). It has to be highlighted here that patients in such series likely represent a selected subpopulation, as mutational analysis is not performed in all patients, e.g. in the case of early, operable disease. The true incidence of $P D G$ FRA-mutated GISTs likely varies between different subpopulations of patients (e.g. dependent on the anatomical site of the primary tumor). It should also be considered that a PDGFRA-mutated GIST is believed to have a less aggressive natural course as compared to other GIST genotypes. In a large European adjuvant GIST trial, however, the frequency of p.D842V was as high as 14\% (data unpublished). It should also not be forgotten that GISTs can be caused by other multi-resistant Kit, PDGFRA, or 'wild type' genotypes, as described above [17].

Mutational testing of tumor tissue has become common practice in the context of clinical trials and in high-volume GIST reference centers, but is not uniformly used on a global scale. The indications for mutational analysis are poorly defined. GIST experts in reference centers generally advocate broad genotyping of advanced GIST cases, primarily based on their interest in the disease biology, 
the molecular epidemiology of GISTs, and the mechanisms behind primary and secondary TKI resistance occurring in this specific tumor type.

The European Society for Medical Oncology (ESMO)/European Sarcoma Network Working Group recommended in 2014 the use of genetic testing for known mutations involving Kit and PDGFRA genes, to confirm the diagnosis of GIST in case of diagnostic uncertainty [18]. The Working Group also highlighted that mutational analysis has a certain prognostic and predictive value to assess the sensitivity of an individual tumor to molecular-targeted therapy, so that its inclusion in the diagnostic work-up of all GISTs was recommended as standard practice (with the exception of very small primary non-rectal GISTs). This European group of experts also suggested centralizing mutational analysis in laboratories enrolled in external quality assurance programs and with specific expertise in this rare disease and complex technology [19].

Comparing different healthcare systems and countries, the current clinical use of mutational analysis is highly variable. In some countries, mutational testing is not available. In other regions, genetic testing is readily established but is performed only in a low percentage of patients. Reimbursement of mutational analysis of GIST by health insurances varies from country to country, and in some geographic regions costs for genetic testing may be prohibitive as even the highly efficacious treatment with imatinib is not uniformly available and covered for by insurances.

\section{Materials and Methods}

The current article describes the rationale for genetic testing of GIST tissue, looks at financial implications associated with such analyses and assesses, in a hypothetical manner, potential cost savings introduced by routine mutational testing and tailored use of TKIs based on genotyping. The work is based on epidemiological data, drug costs, reimbursement criteria, and market research figures.

\section{Background Information}

The Kingdom of Belgium has a population of about 11 million people. Healthcare in Belgium is publicly funded and covered by social security nationwide. Expensive drugs such as TKIs are paid for by the national health insurance system for key indications such as GIST. Imatinib is reimbursed up to a dose of $400 \mathrm{mg}$ (orally (p.o.))/day for metastatic disease, and for up to 3 years for adjuvant treatment of high-risk GISTs. Sunitinib can be prescribed and is reimbursed after imatinib failure, and regorafenib has recently become available for routine use.

Generally speaking, Belgium has a somewhat more restrictive policy regarding the reimbursement of anticancer agents than that of some surrounding European countries. To achieve the most rational use of the limited available healthcare resources, the government bodies have taken a very pragmatic approach for reimbursement of imatinib, by indirectly mandating mutational analysis for each patient applying for the use of imatinib. The TKI is only covered by the health insurance if prescribed by a physician who confirms in writing on a specific form that the tumor does not carry the primary resistant $P D G$ FRA mutation p.D842V. This procedure indirectly mandates to perform mutational analysis in GISTs before prescribing the expensive kinase inhibitor. The exclusion of patients with the PDGFRA mutation p.D842V is based on an academic input of the first author of this publication during the reimbursement discussions on the adjuvant use of imatinib. Reimbursement for other mutational GIST variants is, however, not yet regulated.

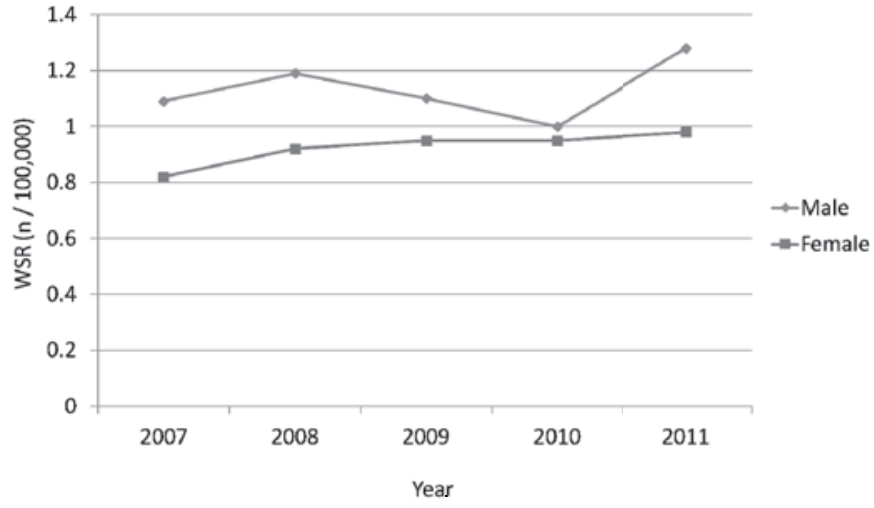

Fig. 1. Incidence of GIST in Belgium, 2007-2011 (Belgian Cancer Registry). $\mathrm{WSR}=$ Age-corrected standardized incidence rate.

\section{Epidemiology of GIST in Belgium}

Every year, about 200 new GIST diagnoses are notified to the Belgian Cancer Registry, corresponding to a crude incidence rate of 1.9 per 100,000 person years. The age standardized incidence rate (World Standard Population) in Belgium over the period 2007 to 2011 is illustrated in figure 1 and is around 1 per 100,000 in both men and women (information provided by the Belgian Cancer Registry) (fig. 1).

\section{Results}

\section{Availability and Cost Burden of Routine Genetic Testing}

Mutational analysis of GIST tissue is available in a number of academic institutions and commercial laboratories in Belgium. Some of these laboratories have participated in the past in GISTspecific quality assurance programs, and at least one of them functioned as central reference laboratory in the context of prospective GIST trials run by pharmaceutical companies.

The public healthcare insurance routinely covers costs of approximately $€ 260$ for both Kit and PDGFRA gene analysis. Some physicians prefer to test for the PDGFRA p.D842V mutation only, which renders the mutational analysis even cheaper, using allelespecific probes as a pragmatic approach. Most GIST experts, however, prefer to have information on the mutational status of both the Kit and PDGFRA genes or even want to see the broader genetic profile, covering rarer variants of the disease. In other European countries commercial providers charge around $€ 500$ for analysis of only 1 gene, but this varies considerably on an international level (personal information).

If all newly diagnosed Belgian GIST patients $(n=200$ per year), independent of specific clinical features, individual prognosis, or risk of relapse of the tumor, had their tissue tested for at least the Kit and PDGFRA mutations, the diagnostic costs for the mutational analysis would be in the range of $€ 52,000$ for the healthcare system. At present, however, most experts are not advocating the genetic testing of all newly diagnosed cases but support a more selective approach, e.g. focusing on subsets of GIST patients at higher risk for an aggressive local behavior, systemic spread, or drug resistance of their disease. This strategy further reduces the cost burden for mutational testing. 
Financial Impact of Adjuvant Imatinib Therapy in Belgium

The annual drug costs of therapy with imatinib in Belgium are approximately $€ 32.000$ per patient in the adjuvant setting. The reimbursed duration of adjuvant treatment with imatinib is 3 years, which theoretically translates into cumulative drug costs for adjuvant therapy of high-risk GIST of $€ 96.000$ per treated patient.

If patients with PDGFRA p.D842V-mutated GIST are not treated with adjuvant imatinib, the expected savings for the healthcare system are $€ 32.000$ per year (or $€ 96.000$ over a typical 3-year treatment period) per patient.

The exact percentage of patients with newly diagnosed GIST who are treated with adjuvant imatinib is unknown. According to global imatinib market research figures (Novartis, personal communication), 22\% of all GIST patients receiving imatinib are treated in an adjuvant fashion for high-risk disease. Let us assume that $50 \%$ of all GIST patients are cured by surgery as single treatment modality, which is a figure often communicated to patients undergoing GIST surgery. The other half of the GIST patients ( $\mathrm{n}=$ 100 per year in Belgium) could potentially qualify for adjuvant and/or palliative (in case of metastasis) imatinib treatment. If $22 \%$ of the imatinib-treated patients on the global scale receive the drug in the postoperative setting for high-risk tumors, and if we assume that these figures were also representative of the Belgian situation, around 22 patients would start adjuvant treatment with imatinib on an annual basis.

Assuming a total number of 22 Belgian GIST patients qualifying every year for adjuvant therapy based on criteria such as tumor size, mitotic index, and anatomical location alone, and considering an incidence of $4-14 \%$ of imatinib-refractory PDGFRA p.D842V mutations (plus other, even rarer imatinib-resistant subtypes), the savings for the healthcare system are $€ 32.000$ per year times 0.88 3.08 patients, which equals $€ 28,160-98,560$ (or $€ 84,480-295,680$ over a typical 3-year treatment period). In total, the savings for avoiding ineffective treatment with imatinib in the adjuvant subset of GIST patients with an imatinib-resistant GIST genotype in Belgium would be in the range of $€ 84,480-295,680$ per year, which is 1.6-5.6 times the cost of performing mutational analysis for the Kit and PDGFRA genes in all GIST patients in Belgium per year, independent of any clinical features. However, testing of all cases, including patients with what is generally called 'micro-GIST', is not common practice and likely not relevant.

\section{Financial Impact of Palliative Imatinib Treatment in Belgium}

According to large prospective clinical trials, the median time to disease progression in patients with metastatic GIST during treatment with imatinib $400 \mathrm{mg}$ is around 2 years [4], but many physicians tend to continue imatinib beyond Response Evaluation Criteria in Solid Tumors (RECIST) progression until a clinically relevant increase in tumor burden occurs; so, the exact duration of imatinib treatment outside of clinical trials is actually unknown. Some exceptional patients with long-term response have now been receiving imatinib for more than 12 years in Belgium without clinically relevant disease progression. Furthermore, many patients continue treatment with a higher daily dose of imatinib (600-800 mg p.o./day) at the point of progression on the 400-mg standard dose. The additional drug dose is not reimbursed by the national health insurance in this country, and physicians and patients are dependent on the compassionate use of free drug from pharmaceutical companies such as Novartis.

The annual direct drug costs of palliative therapy with imatinib $400 \mathrm{mg}$ p.o. in Belgium are approximately $€ 32.000$ per patient in the metastatic, inoperable setting or in the case of a ruptured primary tumor, which is regarded as micrometastatic disease.

If patients with (micro)metastatic PDGFRA p.D842V-mutated GIST were not treated with imatinib, the savings for the healthcare system would in theory be $€ 32.000$ per annum. The percentage of patients diagnosed with metastatic GIST who are treated with imatinib today is likely in the range of $100 \%$. Imatinib is perceived by physicians and patients as a very well-tolerated agent, and firstline trials with other, experimental, agents are currently unavailable. Physicians tend to give imatinib as first-line treatment irrespective of the presence or absence of mutational information. Even in the case of a documented imatinib-insensitive mutation, many patients will still be treated with prescription imatinib in the absence of treatment alternatives and in the hope that the patient will derive some benefit from the well-tolerated agent. First-line use of other TKIs is not a common practice and not in line with current reimbursement criteria, at least in Belgium, so physicians cannot avoid starting treatment with imatinib.

As described above, a GIST driven by a PDGFRA mutation generally tends to have a different natural course than a GIST with primary Kit mutation. These GIST variants have a more indolent spontaneous course, but tend to be more resistant to drug treatment. If a patient with metastatic PDGFRA p.D842V GIST starts imatinib for the non-scientific, reimbursement-driven reasons described above, it is not unlikely that such a patient remains on palliative treatment for several months or even a few years without clinically relevant progression. The authors of this article follow a number of patients with metastatic, PDGFRA-mutated disease without any specific intervention over such prolonged periods of time. The time to spontaneous progression or imatinib failure in such patients is not well documented, but a conservative median 6-month interval can be assumed.

Assuming a total number of 100 Belgian GIST patients qualifying every year for palliative therapy (50\% of the annual incidence), and considering an incidence of $4-14 \%$ of imatinib-refractory PDGFRA p.D842V mutations, the savings for the healthcare system would be $€ 32,000$ per year times $4-14$ patients. Considering a mean treatment duration of 6 months, these estimates would translate into annual savings for the insurance system in case of non-treatment of $€$ 64,000-224,000, which is 1.2-4.3 times the annual cost for performing Kit and PDGFRA mutational analysis in all patients diagnosed with GIST on a yearly basis. Again, routine mutational testing for all patients diagnosed with GIST is not yet common practice.

\section{Global Impact}

Based on figures provided by Novartis (personal communication), 70,328 GIST patients were actively treated with imatinib in 
2014 , including 38,080 patients ( $54 \%$ of the total treated population) with metastatic disease and 22,441 patients (32\%) in the adjuvant setting. Among the latter group of patients, 15,414 patients (22\%) had resected high-risk GISTs. It is unknown in how many imatinib-treated patients mutational analysis is performed and at what point in time. Even in countries with very advanced healthcare systems and availability of the required infrastructure to do mutational analysis without major logistic hurdles, the routine use of mutational testing is very limited, as in the case of the USA.

If we assume a $10 \%$ rate of mutational testing among all TKItreated patients, as reported from some countries, and if we consider an incidence of $2-4 \%$ of PDGFRA p.D842V-mutated GISTs (not taking into account other imatinib-insensitive genotypes) among the treated population, we must accept that at least 1,4072,813 GIST patients are treated on an annual basis with an expensive drug that will likely not change the natural course of the disease of the individual patient. Based on the Belgian price tag for imatinib $400 \mathrm{mg}$ p.o./day, this would imply a waste of considerable financial resources in the range of $€ 45-90$ million per year on a global scale.

\section{Discussion}

The early identification of GIST patients in whom imatinib treatment would not be effective in either the adjuvant or the metastatic setting, e.g. by early routine mutational analysis of the Kit and PDGFRA genes, can potentially lead to considerable cost savings, as illustrated by the hypothetical impact of restricting the use of imatinib to patients with proven absence of the PDGFRA p.D842V resistance mutation based on Belgian drug reimbursement criteria.

Not treating such patients is not only 'healthy' for the healthcare system, but also prevents patients from being exposed to potential toxicity, especially in the case of drugs for which long-term adverse effects, even though unlikely, are yet unknown as they have been in clinical use for less than 2 decades.

The financial resources saved by such an approach should ideally be used to provide funding for research on alternative treatment strategies exploring how to overcome the inherent resistance of some genetically defined GIST subsets to established agents, or to make essential drugs such as imatinib, sunitinib, and regorafenib available to underprivileged GIST patients without insurance coverage.

Based on the hypothetical assumptions discussed here, the pragmatic Belgian solution of mandating mutational testing whenever prescribing imatinib for GIST provides immediate savings to the healthcare system. Assessing the true impact of such measures would have to be based on a thorough analysis of the molecular epidemiology of GISTs in different risk strata of this disease and would require a prospective collection of healthcare resource utilization data.

\section{Acknowledgement}

The authors acknowledge logistic support by Nicole Jackson and John Mitchell from InteGro-MC, Hoboken, NJ, USA, who were supported by Bayer.

\section{Disclosure Statement}

P.S. received financial support for GIST research from Novartis and Bayer and received GIST-related honoraria for advisory and educational activities from Novartis and Bayer. M.D.-R. received honoraria for educational activities from Novartis. O.S. received no financial support for GIST research from any pharmaceutical company, but received honoraria for health economic educational activities from several companies. L.v.E. has no conflict of interest. A.W. received honoraria for educational activities from Novartis.

\section{References}

1 Corless CL, Barnett CM, Heinrich MC: Gastrointestinal stromal tumours: origin and molecular oncology. Nat Rev Cancer 2011;11:865-878

2 Barnett CM, Corless CL, Heinrich MC: Gastrointestinal stromal tumors: molecular markers and genetic subtypes. Hematol Oncol Clin North Am 2013;27: 871-888.

3 Demetri GD, von Mehren M, Blanke CD, Van den Abbeele AD, Eisenberg B, Roberts PJ, et al.: Efficacy and safety of imatinib mesylate in advanced gastrointestinal stromal tumors. N Engl J Med 2002;347:472-480.

4 GIST Meta-Analysis Group: Comparison of two doses of imatinib for the treatment of unresectable or metastatic gastrointestinal stromal tumors: a meta-analysis of 1640 patients. J Clin Oncol 2010;28:1247-1253.

5 Liegl B, Kepten I, Le C, Zhu M, Demetri GD, Heinrich $\mathrm{MC}$, et al.: Heterogeneity of kinase inhibitor resistance mechanisms in GIST. J Pathol 2008;216:64-74.
6 6 Demetri GD, van Oosterom AT, Garrett CR, Blackstein ME, Shah MH, Verweij J, McArthur G, Judson IR, Heinrich MC, Morgan JA, Desai J, Fletcher CD, George S, Bello CL, Huang X, Baum CM, Casali PG: Efficacy and safety of sunitinib in patients with advanced gastrointestinal stromal tumour after failure of imatinib: a randomised controlled trial. Lancet 2006; 368:1329-1338.

7 Gramza AW, Corless CL, Heinrich MC: Resistance to tyrosine kinase inhibitors in gastrointestinal stromal tumors. Clin Cancer Res 2009;15:7510-7518.

$>$ Heinrich MC, Maki RG, Corless CL, Antonescu CR, Harlow A, Griffith D, et al.: Primary and secondary kinase genotypes correlate with the biological and clinical activity of sunitinib in imatinib-resistant gastrointestinal stromal tumor. J Clin Oncol 2008;26:53525359. 9 Demetri GD: Differential properties of current tyros-
ine kinase inhibitors in gastrointestinal stromal tumors. Semin Oncol 2011;38(suppl 1):S10-S19.
10 Debiec-Rychter M, Cools J, Dumez H, Sciot R, Stul M, Mentens N, et al.: Mechanisms of resistance to imatinib mesylate in gastrointestinal stromal tumors and activity of the PKC412 inhibitor against imatinibresistant mutants. Gastroenterology 2005;128:270-279.

11 Corless CL, Schroeder A, Griffith D, Town A, McGreevey L, Harrell P, et al.: PDGFRA mutations in gastrointestinal stromal tumors: frequency, spectrum and in vitro sensitivity to imatinib. J Clin Oncol 2005;23: 5357-5364.

12 Debiec-Rychter M, Sciot R, Le Cesne A, Schlemmer M, Hohenberger P, et al.: Kit mutations and dose selection for imatinib in patients with advanced gastrointestinal stromal tumours. Eur J Cancer 2006;42:1093-1103.

13 Wozniak A, Rutkowski P, Schöffski P, Ray-Coquard I, Hostein I, Schildhaus HU, et al.: Tumor genotype is an independent prognostic factor in primary gastrointestinal stromal tumors of gastric origin: a European multicenter analysis based on ConticaGIST. Clin Cancer Res 2014;20:6105-6116. 
14 Pantaleo MA, Nannini M, Corless CL, Heinrich MC Quadruple wild-type (WT) GIST: defining the subse of GIST that lacks abnormalities of Kit, PDGFRA, SDH, or Ras signaling pathways. Cancer Med 2015;4: 101-103.

15 Joensuu H, Vehtari A, Riihimäki J, Nishida T, Steigen $\mathrm{SE}$, Brabec $\mathrm{P}$, et al.: Risk of recurrence of gastrointesti nal stromal tumour after surgery: an analysis of pooled population-based cohorts. Lancet Oncol 2012;13:265274.
6 Cassier PA, Fumagalli E, Rutkowski P, Schöffski P, Van Glabbeke M, Debiec-Rychter M, et al.: Outcome of patients with platelet-derived growth factor receptor alpha-mutated gastrointestinal stromal tumors in the tyrosine kinase inhibitor era. Clin Cancer Res 2012;18: 4458-4464.

17 Serrano C, Wang Y, Mariño-Enríquez A, Lee JC, Ravegnini G, Morgan JA, et al.: KRAS and Kit gatekeeper mutations confer polyclonal primary imatinib resistance in GI stromal tumors: relevance of concomitant phosphatidylinositol 3-kinase/Akt dysregulation. J Clin Oncol 2015;33:e93-e96.
18 The ESMO/European Sarcoma Network Working Group Gastrointestinal Stromal Tumours: ESMO clinical practice guidelines for diagnosis, treatment and follow-up. Ann Oncol 2014;25(suppl 3):iii21-iii26.

9 Hostein I, Debiec-Rychter M, Olschwang S, Bringuier PP, Toffolati L, Gonzalez D, et al.: A quality control program for mutation detection in Kit and PDGFRA in gastrointestinal stromal tumours. J Gastroenterol 2011; 46:586-594. 